\title{
Novel Two-Dimensional Silica Monolayers with Tetrahedral and Octahedral Configurations
}

\author{
Gaoxue Wang, ${ }^{\dagger}$ G. C. Loh, ${ }^{\dagger}+$ Ravindra Pandey, ${ }^{* \dagger}$ and Shashi P. Karna ${ }^{\S}$ \\ ${ }^{\dagger}$ Department of Physics, Michigan Technological University, Houghton, Michigan 49931, United States \\ ${ }^{\ddagger}$ Institute of High Performance Computing, 1 Fusionopolis Way, \#16-16 Connexis, Singapore 138632 \\ ${ }^{\S}$ U.S. Army Research Laboratory, Weapons and Materials Research Directorate, ATTN: RDRL-WM, Aberdeen Proving Ground, \\ Maryland 21005-5069, United States
}

\section{Supporting Information}

ABSTRACT: Freestanding and well-ordered two-dimensional (2D) silica monolayers with tetrahedral ( $\mathrm{T}$-silica) and octahedral ( $\mathrm{O}$-silica) building blocks are found to be stable by first-principles calculations. T-silica is formed by corner-sharing $\mathrm{SiO}_{4}$ tetrahedrons in a rectangular network, and $\mathrm{O}$-silica consists of edge-sharing $\mathrm{SiO}_{6}$ octahedrons. Moreover, the insulating $\mathrm{O}$-silica is the strongest silica monolayer, and can therefore act as a supporting substrate for nanostructures in sensing and catalytic applications. Nanoribbons of $T$-silica are metallic, while those of $O$-silica have band gaps regardless of the chirality and width. We find the interaction of $O$-silica with graphene to be weak, suggesting the possibility of its use as a monolayer dielectric material for graphene-based devices. Considering that the sixfold-coordinated silica exists at high pressure in the bulk phase, the prediction of a small energy difference of $O$-silica with the synthesized silica bilayer, together with the thermal stability at 1000 $\mathrm{K}$, suggests that synthesis of $\mathrm{O}$-silica can be achieved in experiments.

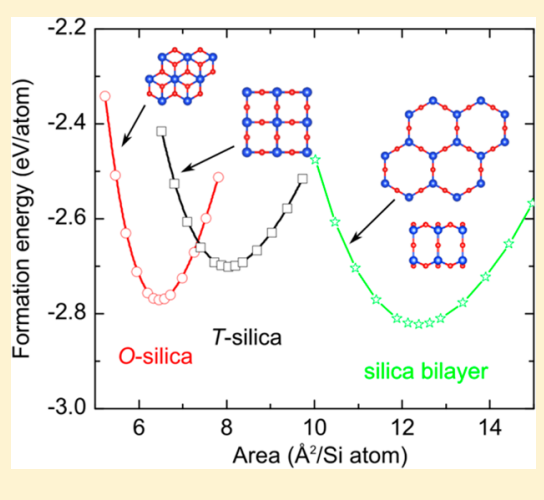

\section{INTRODUCTION}

Silica $\left(\mathrm{SiO}_{2}\right)$ is one of the most abundant materials in Earth's crust. Bulk silica has a rich phase diagram which includes $\alpha$ - and $\beta$-quartz, $\alpha$ - and $\beta$-cristobalite, tridymite, and stishovite. ${ }^{1}$ In general, silica prefers $\mathrm{SiO}_{4}$ tetrahedral unit at ambient conditions consisting of fourfold-coordinated $\mathrm{Si}$ at the center and twofold-coordinated $\mathrm{O}$ at the corners. ${ }^{1,2}$ At high pressure, the phases formed by the $\mathrm{SiO}_{6}$ octahedral unit with sixfoldcoordinated $\mathrm{Si}$ at the center and threefold-coordinated $\mathrm{O}$ at the corners are observed. ${ }^{3-6}$ Stishovite silica with the octahedral unit is thermodynamically stable above $7 \mathrm{GPa}^{4,5,7}$ and is the densest among the allotropes of silica due to its compact structure.

Silica films spontaneously form on a clean silicon surface when the surface is exposed to air. Such two-dimensional (2D) silica films have found their importance in several applications, such as dielectric layers in integrated circuits and supporting substrates for catalysis. ${ }^{8}$ However, their amorphous nature limits the dielectric properties, ${ }^{9}$ and blocks the fabrication of continuous interfaces formed by the dielectric layer with other materials. ${ }^{10,11}$ Therefore, from an application's perspective, well-ordered and freestanding $2 \mathrm{D}$ silica has great advantages over amorphous films. In particular, the monolayer crystalline silica is a much sought after $2 \mathrm{D}$ material as an ideal dielectric for the van der Waals (vdW) heterostructures. ${ }^{10,12}$

Following the stability criterion of the bulk form, 2D silica consisting of $\mathrm{SiO}_{4}$ tetrahedrons has recently been investigated. Silicatene with the $\mathrm{SiO}_{2.5}$ stoichiometry is realized on the $\mathrm{Mo}(112)$ surface. ${ }^{8,13}$ It is formed by corner-sharing $\mathrm{SiO}_{4}$ tetrahedrons in a hexagonal pattern. One of the oxygen atoms in each tetrahedron is bonded strongly to the Mo surface through the $\mathrm{Si}-\mathrm{O}-\mathrm{Mo}$ link, thus limiting the transferability of silicatene. Also, a bilayer silica has been successfully deposited on $\mathrm{Ru}(0001)$ surface with corner-sharing tetrahedrons. ${ }^{14}$ It has the $\mathrm{SiO}_{2}$ stoichiometry, and is weakly anchored on the metal surface due to vdW interactions. ${ }^{9,14,15}$ Such ultrathin bilayers, which could possibly be peeled off from the substrate, are now being explored by experiments. ${ }^{14,16-18}$ On the theoretical front, structural and electronic properties of $\alpha$-silica with alternating $\mathrm{sp}^{3}$ and $\mathrm{sp}^{2}$ bonds have been reported recently. ${ }^{19}$ In spite of the extensive experimental efforts, freestanding and transferable monolayer silica has not yet been realized and the monolayer configuration beyond the tetrahedral building blocks has seldom been reported. ${ }^{19-21}$ There also appears to be a lack of fundamental understanding about the stability, electronic structure, and suitability of silica monolayers as a noninteracting dielectric in vdW electronics.

Considering the importance of freestanding $2 \mathrm{D}$ oxides as potential dielectric layers in nanoelectronic devices ${ }^{10,22}$ and a wide range of other applications, such as photonics and optoelectronics, ${ }^{23}$ we have investigated the possible freestanding silica monolayers together with their stability, electronic band structure, and mechanical properties by the use of density functional theory (DFT) and particle swarm

Received: February 17, 2015

Revised: May 11, 2015

Published: May 20, 2015 
optimization (PSO). We have also investigated the electronic band structure of silica "nanoribbons" with armchair and zigzag edges. For the $2 \mathrm{D}$ silica, we have calculated and reported scanning tunneling microscope (STM) images. Our calculations show that the $2 \mathrm{D}$ silica monolayer with the $\mathrm{SiO}_{4}$ tetrahedral structure in a rectangular network ( $T$-silica) is stable. More importantly, we find that a $2 \mathrm{D}$ silica with the octahedral configuration ( $O$-silica) is also stable, and is more compact and stronger than the previously reported monolayers including $\alpha$-silica and silicatene. ${ }^{13,19}$ Finally, in order to assess the suitability of the $2 \mathrm{D}$ silica in nanoelectronics, we have calculated the equilibrium configuration, the stability, and the interaction between (1) two $O$-silica monolayers and (2) a graphene monolayer and an $\mathrm{O}$-silica monolayer. Our calculations show a rather weak (vdW type) interaction between graphene and $O$-silica monolayers, suggesting the applicability of the latter as a dielectric in graphene-based nanoelectronics.

\section{COMPUTATIONAL METHODS}

The stable configurations of the $2 \mathrm{D}$ silica structures were obtained using the PSO methodology as implemented in the CALYPSO program package. ${ }^{24-27}$ Constraining the stoichiometry to be $\mathrm{SiO}_{2}$, the given configuration is optimized which, in the next cycle (i.e., generation), surmounts an energy barrier to arrive at a lower-energy configuration following the PSO method. In our calculations, the number of configurations (e.g., population) that is produced in each step was set to 24 , and the number of CALYPSO steps (e.g., generation) was fixed to 30 . The history of structural search in terms of energy of each structure in each generation finds $2 \mathrm{D}$ configurations with a rectangular network (i.e., $T$-silica) and an octahedral building block (i.e., $O$-silica) as the energetically preferred monolayer configurations besides other configurations with complex silica networks (see Supporting Information, Figures S1-S3).

The energetically preferred configurations were then fully relaxed to determine their equilibrium structural and electronic properties. The norm-conserving Troullier-Martins pseudopotential $^{28}$ and the Perdew-Burke-Ernzerhof $(\mathrm{PBE})^{29}$ exchange correlation functional form of the DFT together with double- $\zeta$ plus polarization function basis sets were used. The energy convergence was set to $10^{-5} \mathrm{eV}$. The mesh cutoff energy was chosen to be $500 \mathrm{Ry}$. The geometry optimization was considered to be converged when the residual force on each atom was smaller than $0.01 \mathrm{eV} / \AA$. The reciprocal space was sampled by a grid of $(11 \times 11 \times 1) k$ points in the Brillouin zone. In the periodic supercell approach, the vacuum distance normal to the plane was larger than $20 \AA$ to eliminate the interaction between the replicas. The phonon dispersion calculation was based on Vibra of SIESTA utility. ${ }^{30}$

To benchmark our modeling elements, we first considered the cases of $\alpha$-silica $\left(\mathrm{Si}_{2} \mathrm{O}_{3}\right)$ monolayer and silicatene $\left(\mathrm{Si}_{2} \mathrm{O}_{5}\right)$ for calculations. Our calculated bond lengths of $1.83 \AA$ (for sp ${ }^{3}$ bond) and $1.62 \AA$ (for $\mathrm{sp}^{2}$ bond) for $\alpha$-silica are in reasonably good agreement with the corresponding values of 1.76 and 1.58 $\AA$ obtained as previously reported using PBE-DFT level of theory $^{19}$ (see Supporting Information, Figure S4). Our calculated short and long $R_{\mathrm{Si}-\mathrm{O}}$ bonds for silicatene are calculated to be 1.65 and $1.72 \AA$, respectively. The calculated lattice constant of $5.23 \AA$ for silicatene is in excellent agreement with the corresponding experimental value of 5.2-5.4 $\AA^{13}$ Note that $R_{\mathrm{Si}-\mathrm{O}}$ in the bulk $\alpha$-quartz is reported to be $1.61 \AA^{31}$

\section{RESULTS AND DISCUSSION}

Next, monolayers of $T$ - and $O$-silicas were considered to determine their energetic stabilities relative to the silica bilayer, which has been synthesized recently. ${ }^{14}$ Figure 1 shows the

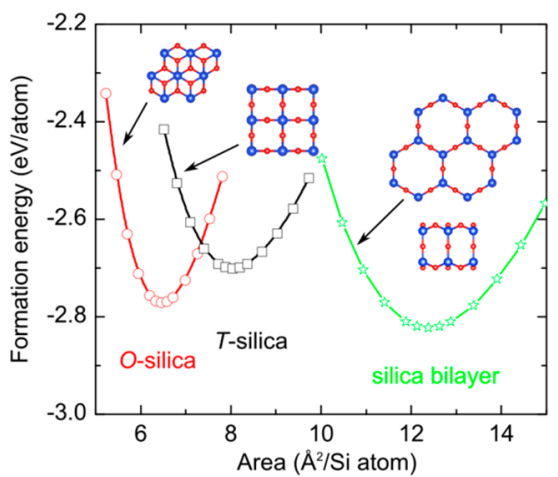

Figure 1. Calculated formation energy vs area/Si atom for $2 \mathrm{D}$ silica configurations.

variation of the formation energy with area per Si atom for silica bilayer, $O$-silica, and T-silica. The formation energy of the $2 \mathrm{D}$ silica configuration with respect to its constituent atoms is calculated as

$$
E_{\text {form }}=\left(E_{\text {total }}-N_{\mathrm{Si}} E_{\mathrm{Si}}-N_{\mathrm{O}} E_{\mathrm{O}}\right) /\left(N_{\mathrm{Si}}+N_{\mathrm{O}}\right)
$$

where $E_{\text {total }}$ is the total energy of the system. $E_{\mathrm{Si}}$ and $E_{\mathrm{O}}$ are the energies of single $\mathrm{Si}$ and $\mathrm{O}$ in bulk $\mathrm{Si}$ and $\mathrm{O}_{2}$ molecule, which are calculated to be -128.80 and $-444.53 \mathrm{eV}$, respectively. $N_{\mathrm{Si}}$ and $N_{\mathrm{O}}$ are the numbers of $\mathrm{Si}$ and $\mathrm{O}$ atoms in the unit cell. The values of $\left(N_{S i}, N_{O}\right)$ are $(1,2),(1,2)$, and $(4,8)$ for $T$-silica, $O$ silica, and silica bilayer, respectively. A negative formation energy suggests the configuration is stable or metastable. ${ }^{32}$ Note that the energy minimum in each curve of Figure 1 represents the equilibrium configuration of the given structure.

The calculated formation energies of $O$-silica and $T$-silica are -2.77 and $-2.70 \mathrm{eV} /$ atom, respectively (Table 1 ). Interestingly, these values are very close to the value of the formation energy of $-2.82 \mathrm{eV} /$ atom for the silica bilayer which has recently been synthesized. ${ }^{14}$ Our results therefore suggest that these novel silica monolayers can be synthesized. In comparison, the calculated formation energies of $\alpha$-silica and silicatene are -1.94 and $-2.24 \mathrm{eV} /$ atom, respectively (see Supporting Information, Figure S5).

$T$-silica is constituted by $\mathrm{SiO}_{4}$ tetrahedrons in a rectangular network (Figure 2). The corner $\mathrm{O}$ atom is shared by the neighboring tetrahedrons. $R_{\mathrm{Si}-\mathrm{O}}$ is $1.68 \AA$ and $\angle \mathrm{O}-\mathrm{Si}-\mathrm{O}$ is $106.9^{\circ}$ in the equilibrium configuration of $T$-silica. Note that $\angle \mathrm{O}-\mathrm{Si}-\mathrm{O}$ for a perfect tetrahedron is $109.5^{\circ}$. The absence of imaginary vibrational frequencies in the calculated phonon dispersion curves (Figure 2c) implies that $T$-silica is dynamically stable. The speed of sound is $\sim 1.9 \mathrm{~km} / \mathrm{s}$ obtained by the slope of the longitudinal acoustic (LA) branch near $\Gamma$. The maximum vibrational frequency of the optical branch is $1015 \mathrm{~cm}^{-1}$. The deformation charge density plot shown in Figure $2 \mathrm{~b}$ suggests the charge transfer from $\mathrm{Si}$ to $\mathrm{O}$ yielding the charge of $\mathrm{Si}$ to be $3.52 e$ as per Mulliken charge analysis. Therefore, the $\mathrm{Si}-\mathrm{O}$ bond retains partial ionic character in $T$ silica. The valence band maximum (VBM) at $\mathrm{X}^{\prime}$ is dominated by $\mathrm{O}-\mathrm{p}$ orbitals and the conduction band minimum (CBM) at $\Gamma$ is composed of Si-s and O-p orbitals as shown by the orbital- 
Table 1. Structural Properties of 2D Silica Structures ${ }^{a}$

\begin{tabular}{lllllc}
\multicolumn{1}{c}{ this work } & \multicolumn{1}{c}{$a_{0}(\AA)$} & $R_{\mathrm{Si}-\mathrm{O}}(\AA)$ & $R_{\mathrm{Si}-\mathrm{Si}}(\AA)$ & $E_{\text {form }}(\mathrm{eV} /$ atom $)$ & -2.70 \\
$T$-silica $\left(\mathrm{SiO}_{2}\right)$ & 2.84 & 1.68 & 2.84 & -2.77 & 159 \\
$\mathrm{O}$-silica $\left(\mathrm{SiO}_{2}\right)$ & 2.73 & 1.84 & 2.73 & -2.82 & 215 \\
silica bilayer $\left(\mathrm{Si}_{4} \mathrm{O}_{8}\right)$ & $5.35(5.40)^{16}$ & $1.65(1.67)^{18}$ & $3.09(3.12)^{18}$ & 154
\end{tabular}

$a^{a} a_{0}$ is the lattice constant, $R_{\mathrm{Si}-\mathrm{O}}$ is the near-neighbor distance, $R_{\mathrm{Si}-\mathrm{Si}}$ is the distance between $\mathrm{Si}$ atoms, $E_{\mathrm{form}}$ is the formation energy defined by eq 1 , and $C$ is the in-plane stiffness. For silica bilayer, the experimental values are given in parentheses.

(a)

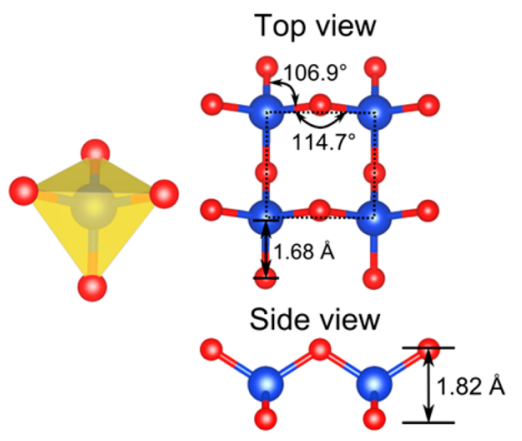

(b)

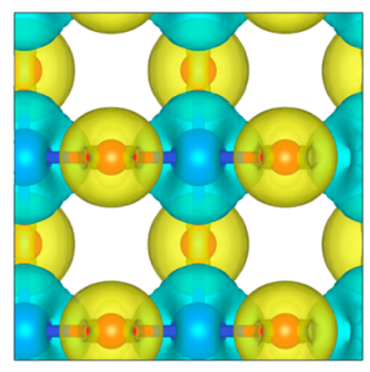

(c)

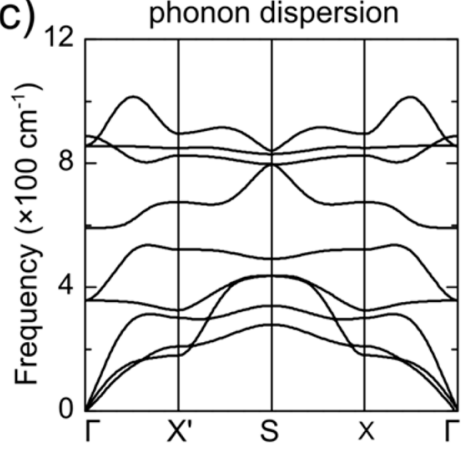

(d)

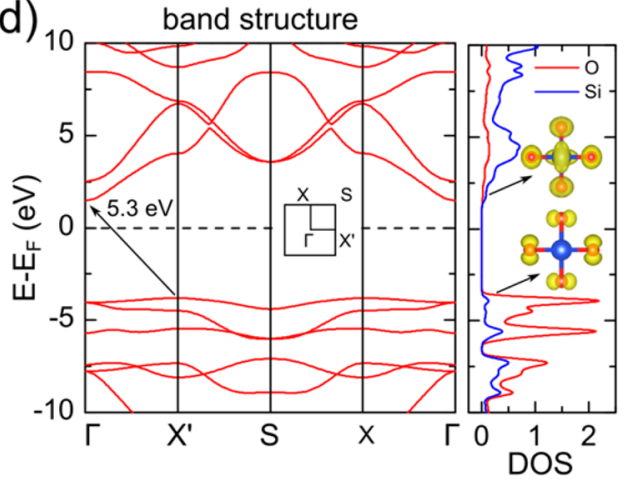

Figure 2. T-silica. (a) Atomic structure. (b) Deformation electron density (with isosurface of $0.01 \mathrm{e} / \AA^{3}$ ). Blue represents depletion of electrons, and yellow represents accumulation of electrons. (c) Phonon dispersion curves and (d) electronic band structure and DOS. Inset show the charge density at VBM and CBM. The high symmetry points are $\Gamma(0,0,0), X^{\prime}(1 / 2,0,0), S(1 / 2,1 / 2,0)$, and $X(0,1 / 2,0)$ in reciprocal lattice.

projected density of states (DOS) in Figure $2 \mathrm{~d}$. $T$-silica has an indirect $\left(\mathrm{X}^{\prime} \rightarrow \Gamma\right)$ band gap of $5.3 \mathrm{eV}$, whereas the direct gap at $\Gamma$ is $5.6 \mathrm{eV}$.

Unlike silica bilayer and $T$-silica, the basic building blocks of $\mathrm{O}$-silica are the $\mathrm{SiO}_{6}$ octahedron as shown in Figure 3. Each $\mathrm{Si}$ is surrounded by six $\mathrm{O}$ atoms forming an octahedron. $\mathrm{O}$-silica has a structure similar to that of the metallic $1 \mathrm{~T}$ phase of $\mathrm{MoS}_{2} ;{ }^{33}$ the Si layer is sandwiched between the top and bottom $\mathrm{O}$ layers. It also has the appearance of a centered honeycomb (top view of Figure 3a). Our calculated $R_{\mathrm{Si}-\mathrm{O}}$ is $1.84 \AA$, which is slightly larger than the measured value of the axial $\mathrm{Si}-\mathrm{O}$ bond length of $1.81 \AA$ in stishovite. ${ }^{34}$ The $\angle \mathrm{O}-\mathrm{Si}-\mathrm{O}$ bond angles are calculated to be 83.9 and $96.1^{\circ}$, which are distorted from those $\left(\approx 90^{\circ}\right)$ in a perfect octahedron. Each $\mathrm{O}$ is threefoldcoordinated, with three $\mathrm{Si}-\mathrm{O}$ bonds not lying in the same plane, and $\angle \mathrm{Si}-\mathrm{O}-\mathrm{Si}$ is $96.1^{\circ}$.

The phonon dispersion curves show the dynamical stability of $O$-silica (Figure 3c). The speed of sound is $2.3 \mathrm{~km} / \mathrm{s}$ obtained from the slope of the LA branches near $\Gamma$. The maximum vibrational frequency of the optical branch is 912 $\mathrm{cm}^{-1}$, which is smaller than that in $T$-silica, suggesting that the $\mathrm{Si}-\mathrm{O}$ bond is slightly weaker in $\mathrm{O}$-silica. This is consistent with the order of $R_{\mathrm{Si}-\mathrm{O}}$ as the structure changes from $T$-silica (1.68 $\AA$ ) to $O$-silica $\left(1.84 \AA\right.$ ). For the bulk phase, $R_{\mathrm{Si}-\mathrm{O}}$ also increases from the tetrahedral phase to the high-pressure octahedral phase. $^{2}$

The deformation charge density distribution shows the sixfold-coordinated character of Si (Figure 3b). Mulliken charge analysis shows the $\mathrm{Si}$ charge to be $3.49 e$, which is fractionally larger than that of fourfold-coordinated $\mathrm{Si}$ in T-silica. The VBM is dominated by O-p orbitals, and CBM is composed of both $\mathrm{Si}$ $\mathrm{s}$ and O-p orbitals (Figure 3d). Note that the location of VBM is away from $\Gamma$. The calculated band gap is indirect $(\Gamma-\mathrm{M} \rightarrow$ $\Gamma$ ) with a value of $\sim 6.2 \mathrm{eV}$, and the direct band gap at $\Gamma$ is 6.7 $\mathrm{eV}$.

The thermal stabilities of $O$-silica and $T$-silica are further examined by ab initio molecular dynamics (MD) simulations (Figure 4). The MD simulations are based on the Nose thermostat at $1000 \mathrm{~K}^{35}$ We used a large supercell of $5 \times 5$ to minimize the constraints induced by periodicity in the slab model. The averages of the total energies for the structures remain nearly constant during the simulation, and the structures remain unchanged after 5 ps, which suggest that $O$ - and $T$-silicas are thermally stable.

In order to understand and establish the mechanical properties of $2 \mathrm{D}$ silica structures, we calculated the in-plane stiffness, ${ }^{36-38} \mathrm{C}=\left(1 / S_{0}\right)\left(\partial^{2} E_{\mathrm{S}} / \partial \varepsilon^{2}\right)$. Here, $S_{0}$ is the equilibrium area of the structure, $E_{S}$ is the strain energy, defined as the energy difference between the strained and the relaxed 
(a)

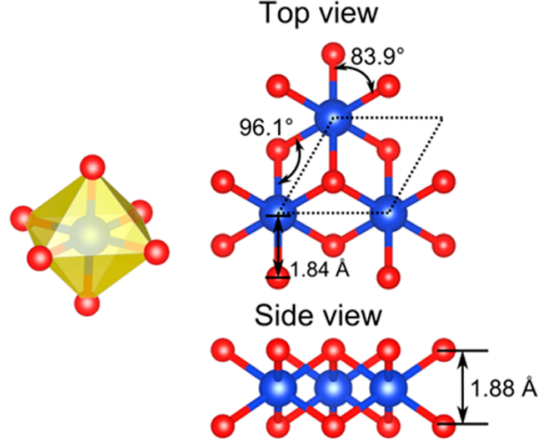

(b)

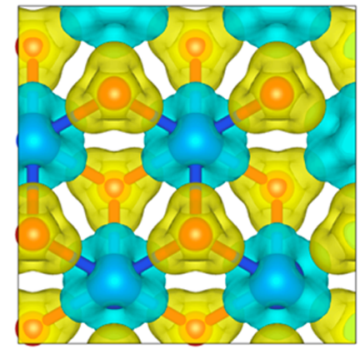

(c)

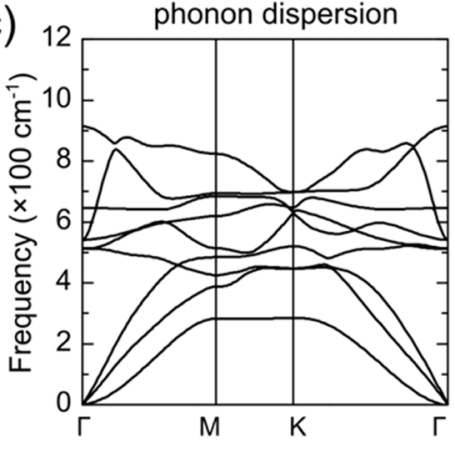

(d)

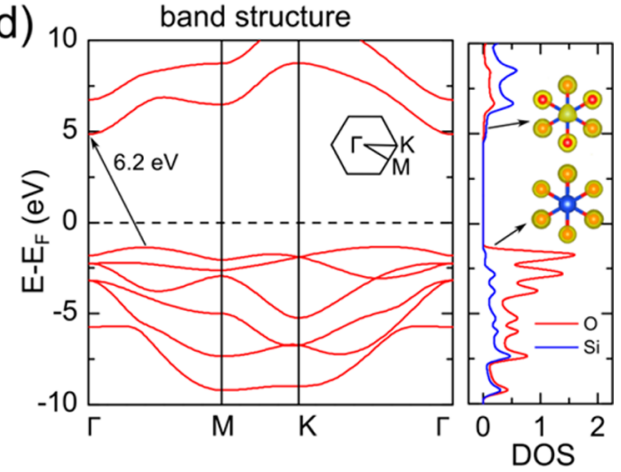

Figure 3. O-silica. (a) Atomic structure. (b) Deformation electron density (with isosurface of $0.01 \mathrm{e} / \AA^{3}$ ). Blue represents depletion of electrons, and yellow represents accumulation of electrons. (c) Phonon dispersion curves and (d) electronic band structure and DOS. Inset shows the charge density at VBM and CBM. The high symmetry points are $\Gamma(0,0,0), \mathrm{K}(2 / 3,1 / 3,0)$, and $\mathrm{M}(1 / 2,0,0)$ in reciprocal lattice.

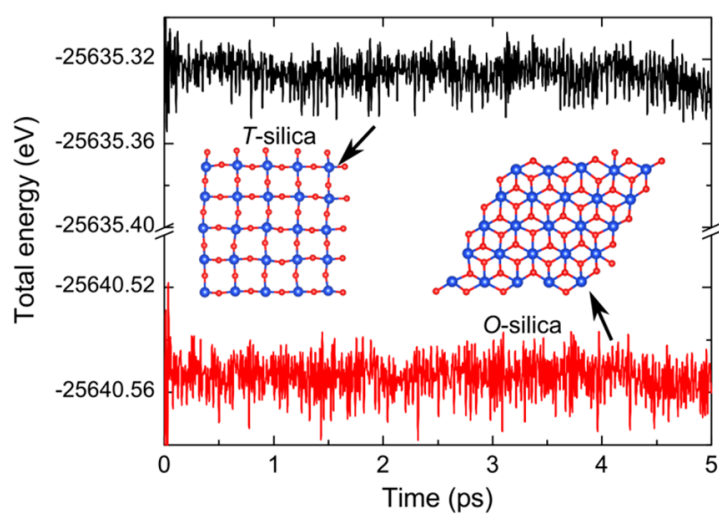

Figure 4. Energy fluctuations of $T$ - and $O$-silicas as a function of simulation steps. The insets show the corresponding structueres with the simulation up to 5 ps.

structures, and $\varepsilon$ is the uniaxial strain applied to the structure. In order to establish the reliability of our approach, we first calculated the in-plane stiffness for graphene. The calculated value $C$ for graphene is $342 \mathrm{~N} / \mathrm{m}$, which is in excellent agreement with the corresponding experimental value of $340 \pm$ $40 \mathrm{~N} / \mathrm{m},{ }^{39}$ giving confidence in our calculated values of $C$ for $2 \mathrm{D}$ silica. Our calculations show that $\mathrm{O}$-silica has the largest inplane stiffness of $215 \mathrm{~N} / \mathrm{m}$ among the $2 \mathrm{D}$ silica structures (Table 1). This is in line with $O$-silica being more compact than other 2D silica structures (Figure 1). These results, therefore, suggest that $O$-silica could be freestanding and a good support for applications in catalysis and electronic devices. ${ }^{40}$ In contrast, the stiffness constant values for $\alpha$-silica and silicatene are calculated to be 17 and $45 \mathrm{~N} / \mathrm{m}$, respectively, which are much smaller than that for the $O$-silica, suggesting the former two to be relatively soft compared to the latter.
To get further insight into the stability of sixfold-coordinated silica monolayer, the terms contributing to the total energy are compared in Table 2. A smaller $R_{\mathrm{Si}-\mathrm{Si}}$ leads to the highest ion-

Table 2. Decomposition of the Total Energy of $T$-Silica, $O$ Silica, and Silica Bilayer ${ }^{a}$

\begin{tabular}{llll} 
& \multicolumn{1}{c}{$T$-silica } & \multicolumn{1}{c}{$O$-silica } & silica bilayer \\
kinetic & 217.49 & 218.01 & 219.40 \\
Hartree & 965.44 & 1094.34 & 376.41 \\
exchange correlation & -93.27 & -93.72 & -93.42 \\
ion-electron & -2129.99 & -2391.06 & -953.15 \\
ion-ion & 698.40 & 830.44 & 108.71 \\
total & -341.93 & -342.00 & -342.05
\end{tabular}

${ }^{a}$ The energy is in units of $\mathrm{eV} /$ atom.

ion Coulomb interaction in $\mathrm{O}$-silica. However, the compact electronic arrangement of $\mathrm{Si}$ and $\mathrm{O}$ atoms yields the lowest ion-electron Coulomb energy in $\mathrm{O}$-silica. This interplay of bonding and geometrical arrangements facilitates the overall comparable total energy for $T$-silica, $O$-silica, and silica bilayer. Analysis of the electron density with the quantum theory of "atoms in molecule" via the electron localization function (ELF), charge density, and Laplacian plots shows that the Si-O bond is slightly ionic in nature in the octahedral coordinated silica compared to that in tetrahedral coordinated silica layers (see Supporting Information, Figures S6 and S7).

Next, we performed simulation of the scanning tunneling microscopy (STM) images based on the Tersoff and Hamann approximation $^{41}$ at constant current mode to mimic the experimental setup. ${ }^{42-45}$ A gold cluster, $\mathrm{Au}_{13}$, was used to represent the cap configuration of the STM tip. The calculated STM images are shown in Figure 5. The simulated STM image of the bilayer silica (Figure 5d) is in excellent agreement with 


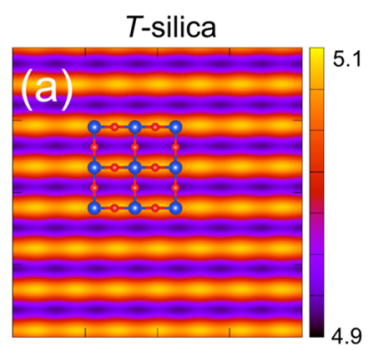

$\alpha$-silica

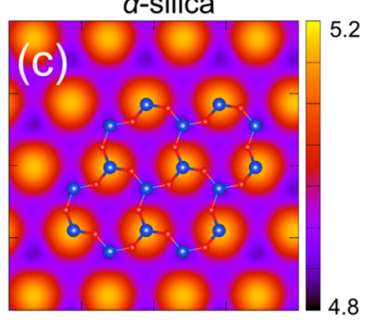

O-silica

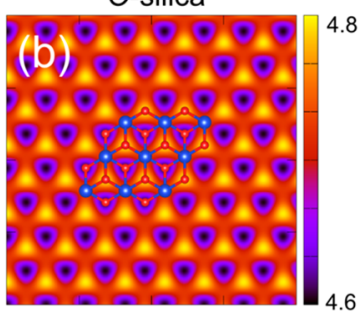

silica bilayer

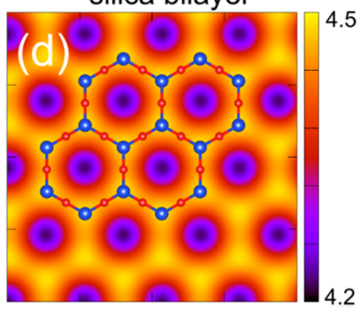

Figure 5. Simulated STM images for 2D silica using constant current mode. The size of the STM images are $20 \AA \times 20 \AA$; the scale bars are in the unit of angstroms.

the corresponding measured STM image, ${ }^{18}$ showing the surface with a hexagonal pattern. The STM image with clear contrast can be obtained at a small bias voltage, and metal substrates do not modify the calculated STM results (see Supporting Information, Figures S8 and S9).

The simulated STM image of the T-silica (Figure 5a), on the other hand, shows a stripe-like surface feature. $O$-silica has triangle-like surface character (Figure $5 \mathrm{~b}$ ) contributed by the top $\mathrm{O}$ atoms, while $\alpha$-silica shows a flat dot-like surface (Figure 5c) due to the $\mathrm{sp}^{2}$ bonding features. We expect that the distinct characteristics of $2 \mathrm{D}$ silica structures will be useful in identifying and distinguishing future experimental STM images of these nanoscale materials.

Integration of the 2D materials into electronic devices can generally be performed by cutting the $2 \mathrm{D}$ sheet into finite components, such as nanoribbons. For $T$-silica, cutting can be done along the rectangular lattice direction (see Supporting Information, Figure S10a). In the equilibrium configuration, we find that atoms near the edges of nanoribbon are reconstructed, and the ribbon is curved laterally with $\angle \mathrm{O}-\mathrm{Si}-\mathrm{O}$ to $109.2^{\circ}$, which is very close to that of a perfect octahedron. $T$-silica nanoribbon is metallic, and the energy band crossing the Fermi level is derived from dangling bonds of the edge $\mathrm{Si}$ atoms (Figure 6a).

For $\mathrm{O}$-silica, cutting along the armchair direction (see Supporting Information, Figure S10b) leads to two kinds of armchair nanoribbons with symmetric (Figure 6b) and asymmetric edges (Figure 6c). If we cut $\mathrm{O}$-silica along the zigzag direction, two kinds of edges terminated by either $\mathrm{O}$ or $\mathrm{Si}$ atoms appeared. Therefore, three kinds of zigzag nanoribbons with edges terminated by $\mathrm{Si}$ atoms ( $\mathrm{Si}-\mathrm{Si}$ edge, Figure 6d), $\mathrm{Si}$ and $\mathrm{O}$ atoms ( $\mathrm{Si}-\mathrm{O}$ edge, Figure $6 \mathrm{e}$ ), and $\mathrm{O}$ atoms (a) T-silica ribbon
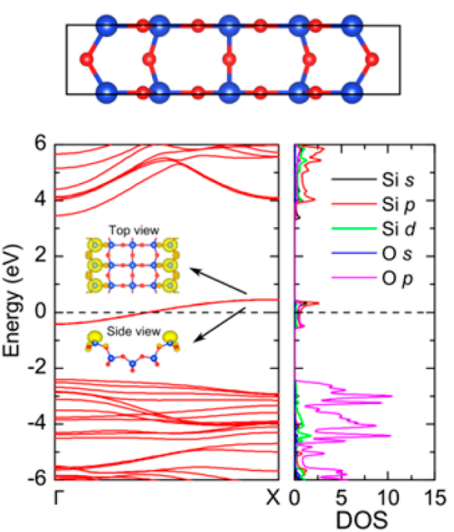

(d) O-silica Si-Si edge zigzag
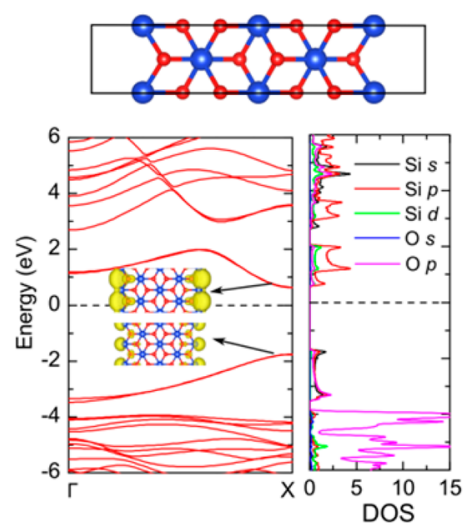

(b)
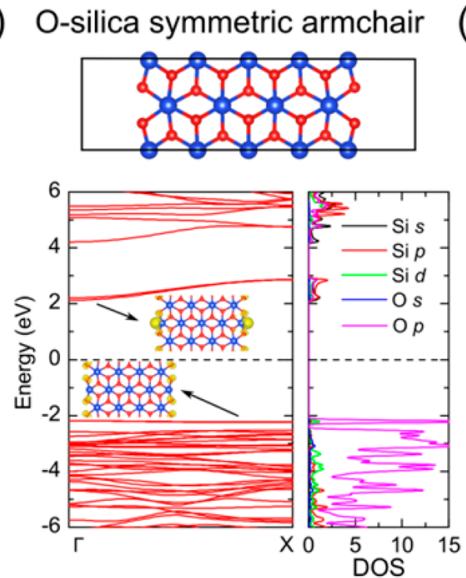

(e)

e) O-silica Si-O edge zigzag
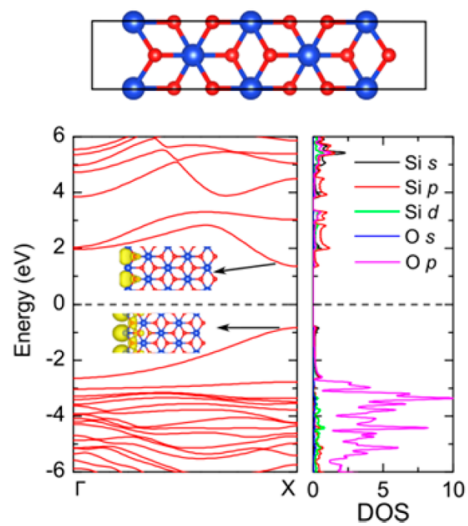

(c) O-silica asymmetric armchair
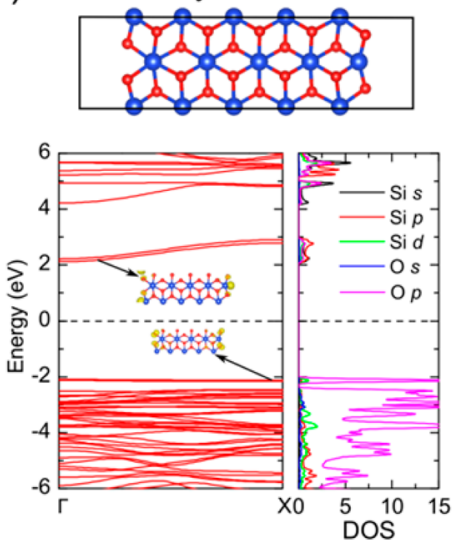

(f) O-silica O-O edge zigzag
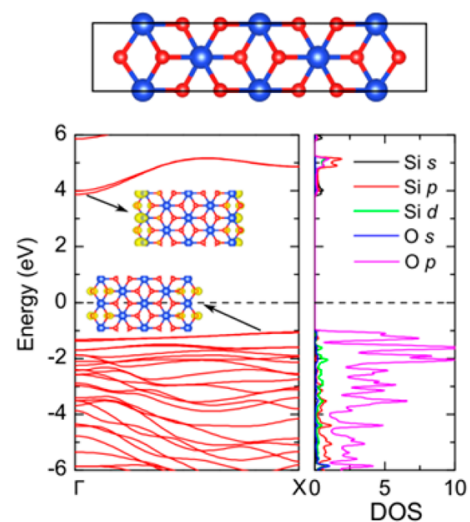

Figure 6. Structure and band structure of (a) $T$-silica nanoribbon and (b)-(f) $O$-silica nanoribbons in the armchair and zigzag configurations. The insets show charge density associated with edge states. 
(O-O edge, Figure 6f) are considered. We find that the nanoribbons considered are stable with minor reconstruction near the edges in their equilibrium configurations. The states associated with the dangling bonds at the edges do not cross the Fermi level, and the band gap is independent of the edge configurations of the $O$-silica nanoribbons. Simulations of $T$ and $O$-silica nanoribbons with larger widths $(\approx 40 \AA)$ confirm that electronic properties of these ribbons are robust regardless of the widths of the ribbons (see Supporting Information, Figure S11).

Finally, in order to address the feasibility of $O$-silica as a dielectric monolayer in the vdW heterostructures, e.g., graphene-based devices, we performed electronic structure calculations, on the graphene/O-silica and the $\mathrm{O}$-silica/O-silica bilayers in the configuration shown in Figure 7. The graphene/ $O$-silica heterostructure was simulated by a supercell of $(2 \times 2)$ $\boldsymbol{R}$ for graphene and $(\sqrt{3} \times \sqrt{3}) \boldsymbol{R}$ for $O$-silica, minimizing the lattice mismatch to $\sim 3 \%$. (a) Graphene/O-silica

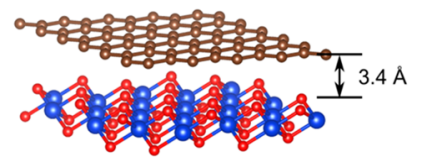

(b)

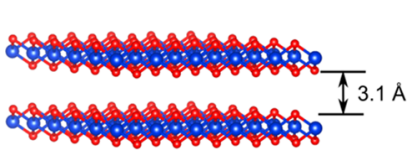

Figure 7. Equilibrium configurations of (a) graphene/O-silica heterostructure and (b) $\mathrm{O}$-silica bilayer.

The vdW correction to the correlation-exchange functional was considered for the heterostructure. ${ }^{46}$ To benchmark the bilayer system, we first considered the bilayer $O$-silica for which the interlayer distance is calculated to be $\sim 3.1 \AA$ with the band gap of $5.6 \mathrm{eV}$. For graphene/O-silica bilayer, the calculated interlayer distance is $\sim 3.4 \AA$ and the binding energy with respect to its constituent monolayers is $24 \mathrm{meV} /$ atom. The results therefore suggest the interaction between graphene and $\mathrm{O}$-silica to be weak, leading to retention of the electronic characteristics of graphene in the heterostructure (see Supporting Information, Figure S12). Interestingly, the band gap in the heterostructure does not open up when we reduce the interlayer distance to $\sim 2.0 \AA$, thus demonstrating that $O$ silica could potentially act as an ideal monolayer insulator in the so-called vdW heterostructure devices.

\section{SUMMARY}

In summary, from our first-principles DFT calculations, silica sheets constituted by either edge-sharing $\mathrm{SiO}_{6}$ octahedrons (i.e., $\mathrm{O}$-silica) or a rectangular network of $\mathrm{SiO}_{4}$ tetrahedrons (i.e., $T$-silica) are found to be stable. Both $2 \mathrm{D}$ silica structures have large in-plane stiffness (mechanical strength) and electrical insulating properties. Additionally, $\mathrm{O}$-silica nanoribbons also have band gaps which are not dependent on their edge configurations, whereas $T$-silica nanoribbons are metallic. The structural simplicity and stiffness thus facilitate the use of freestanding $O$ - and $T$-silica monolayers for versatile applications, e.g., as a single layer insulator in $\mathrm{vdW}$ heterostructures or as a supportive substrate for catalysis.

$O$-silica is the first proposed 2D silica with octahedralcoordinated building blocks, which is of fundamental importance considering that the sixfold-coordinated silica in the bulk phase exists at high pressure. It has been reported that the high-pressure phases of cubic $\mathrm{TiO}_{2}$ and rock salt $\mathrm{GaN}$ could be stabilized in thin films. ${ }^{47,48}$ Also, $2 \mathrm{D}$ materials with similar structure, e.g., $\mathrm{MoS}_{2}{ }^{33}$ have been successfully synthesized via molecular beam epitaxy (MBE) and chemical vapor deposition (CVD) methods. We expect that our results showing a small energy difference of $O$-silica with the synthesized silica bilayer would stimulate and help experimental research to realize $O$ silica in experiments.

\section{ASSOCIATED CONTENT}

\section{S Supporting Information}

Figures S1-S3, history of structural search by CALYPSO; Figure S4, structural and electronic properties of $\alpha$-silica; Figure S5, formation energy of $\alpha$-silica and silicatene; Figure S6, electron localization function (ELF) for bilayer silica, T-silica, and $\mathrm{O}$-silica; Figure S7, 2D charge density contours and Laplacian for silica bilayer, $T$-silica, and $\mathrm{O}$-silica; Figure S8, STM images of freestanding silica bilayer; Figure S9, structure and STM images of bilayer silica on $\mathrm{Ru}(0001)$ surface; Figure $\mathrm{S} 10, T$-silica and $\mathrm{O}$-silica nanoribbons; Figure S11, structure and band structure of $T$-silica and $O$-silica nanoribbons; Figure $\mathrm{S} 12$, electronic structure of $\mathrm{O}$-silica/graphene heterostructure. The Supporting Information is available free of charge on the ACS Publications website at DOI: 10.1021/acs.jpcc.5b01646.

\section{AUTHOR INFORMATION}

\section{Corresponding Author}

*E-mail: pandey@mtu.edu.

\section{Notes}

The authors declare no competing financial interest. E-mail: shashi.p.karna.civ@mail.mil (S.P.K.).

\section{ACKNOWLEDGMENTS}

Helpful discussions with S. Gowtham are acknowledged. RAMA and Superior, high performance computing clusters at Michigan Technological University, were used in obtaining results presented in this paper. The authors appreciate D. R. Banyai for providing the code for STM simulation. This research was partially supported by the Army Research Office through Grant W911NF-14-2-0088.

\section{REFERENCES}

(1) Shaikhutdinov, S.; Freund, H.-J. Ultrathin Silica Films on Metals: The long and winding road to understanding the atomic structure. Adv. Mater. 2013, 25, 49-67.

(2) Demuth, T.; Jeanvoine, Y.; Hafner, J.; Angyan, J. Polymorphism in silica studied in the local density and generalized-gradient approximations. J. Phys.: Condens. Matter 1999, 11, 3833.

(3) Stishov, S.; Belov, N. Crystal structure of a new dense modification of silica $\mathrm{SiO}_{2}$. Dokl. Akad. Nauk SSSR 1962, 143, 951.

(4) Chao, E.; Fahey, J.; Littler, J.; Milton, D. Stishovite, $\mathrm{SiO}_{2}$, a very high pressure new mineral from Meteor Crater, Arizona. J. Geophys. Res. 1962, 67, 419-421.

(5) Teter, D. M.; Hemley, R. J.; Kresse, G.; Hafner, J. High pressure polymorphism in silica. Phys. Rev. Lett. 1998, 80, 2145.

(6) Shieh, S. R.; Duffy, T. S.; Li, B. Strength and elasticity of $\mathrm{SiO}_{2}$ across the Stishovite- $\mathrm{CaCl}_{2}$-type structural phase boundary. Phys. Rev. Lett. 2002, 89, 255507.

(7) Prakapenka, V.; Shen, G.; Dubrovinsky, L.; Rivers, M.; Sutton, S. High pressure induced phase transformation of $\mathrm{SiO}_{2}$ and $\mathrm{GeO}_{2}$ : difference and similarity. J. Phys. Chem. Solids 2004, 65, 1537-1545.

(8) Weissenrieder, J.; Kaya, S.; Lu, J. L.; Gao, H. J.; Shaikhutdinov, S.; Freund, H. J.; Sierka, M.; Todorova, T. K.; Sauer, J. Atomic Structure of a Thin Silica Film on a Mo(112) Substrate: A Two-Dimensional Network of $\mathrm{SiO}_{4}$ Tetrahedra. Phys. Rev. Lett. 2005, 95, 076103. 
(9) Büchner, C.; Lichtenstein, L.; Yu, X.; Boscoboinik, J. A.; Yang, B.; Kaden, W. E.; Heyde, M.; Shaikhutdinov, S. K.; Włodarczyk, R.; Sierka, M. Ultrathin Silica Films: The Atomic Structure of Two-Dimensional Crystals and Glasses. Chem.-Eur. J. 2014, 20, 9176-9183.

(10) Addou, R.; Dahal, A.; Batzill, M. Growth of a two-dimensional dielectric monolayer on quasi-freestanding graphene. Nat. Nanotechnol. 2013, 8, 41-45.

(11) Garces, N.; Wheeler, V.; Hite, J.; Jernigan, G.; Tedesco, J.; Nepal, N.; Eddy, C., Jr.; Gaskill, D. Epitaxial graphene surface preparation for atomic layer deposition of $\mathrm{Al}_{2} \mathrm{O}_{3}$. J. Appl. Phys. 2011, 109, 124304.

(12) Geim, A.; Grigorieva, I. Van der Waals heterostructures. Nature 2013, 499, 419-425.

(13) Yang, B.; Boscoboinik, J. A.; Yu, X.; Shaikhutdinov, S.; Freund, H.-J. Patterned defect structures predicted for graphene are observed on single-layer silica films. Nano Lett. 2013, 13, 4422-4427.

(14) Löffler, D.; Uhlrich, J.; Baron, M.; Yang, B.; Yu, X.; Lichtenstein, L.; Heinke, L.; Büchner, C.; Heyde, M.; Shaikhutdinov, S. Growth and structure of crystalline silica sheet on $\mathrm{Ru}$ (0001). Phys. Rev. Lett. 2010, $105,146104$.

(15) Yang, B.; Kaden, W. E.; Yu, X.; Boscoboinik, J. A.; Martynova, Y.; Lichtenstein, L.; Heyde, M.; Sterrer, M.; Włodarczyk, R.; Sierka, M. Thin silica films on $\mathrm{Ru}$ (0001): monolayer, bilayer and threedimensional networks of $\mathrm{SiO}_{4}$ tetrahedra. Phys. Chem. Chem. Phys. 2012, 14, 11344-11351.

(16) Huang, P. Y.; Kurasch, S.; Alden, J. S.; Shekhawat, A.; Alemi, A. A.; McEuen, P. L.; Sethna, J. P.; Kaiser, U.; Muller, D. A. Imaging atomic rearrangements in two-dimensional silica glass: Watching silica's dance. Science 2013, 342, 224-227.

(17) Huang, P. Y.; Kurasch, S.; Srivastava, A.; Skakalova, V.; Kotakoski, J.; Krasheninnikov, A. V.; Hovden, R.; Mao, Q.; Meyer, J. C.; Smet, J. Direct imaging of a two-dimensional silica glass on graphene. Nano Lett. 2012, 12, 1081-1086.

(18) Lichtenstein, L.; Heyde, M.; Freund, H.-J. Atomic Arrangement in Two-Dimensional Silica: From Crystalline to Vitreous Structures. J. Phys. Chem. C 2012, 116, 20426-20432.

(19) Özçelik, V. O.; Cahangirov, S.; Ciraci, S. Stable Single-Layer Honeycomblike Structure of Silica. Phys. Rev. Lett. 2014, 112, 246803.

(20) Durandurdu, M. Formation of a two-dimensional layered structure in silica under shear stresses: An ab initio study. Phys. Rev. B 2010, 81, 174107.

(21) Wang, R.; Pi, X.; Ni, Z.; Liu, Y.; Lin, S.; Xu, M.; Yang, D. Silicene oxides: formation, structures and electronic properties. Sci. Rep. 2013, 3, 3507.

(22) Robinson, J. A.; LaBella, M.; Trumbull, K. A.; Weng, X.; Cavelero, R.; Daniels, T.; Hughes, Z.; Hollander, M.; Fanton, M.; Snyder, D. Epitaxial Graphene Materials Integration: Effects of Dielectric Overlayers on Structural and Electronic Properties. ACS Nano 2010, 4, 2667-2672.

(23) Ikushima, A. J.; Fujiwara, T.; Saito, K. Silica glass: A material for photonics. J. Appl. Phys. 2000, 88, 1201-1213.

(24) Wang, Y.; Lv, J.; Zhu, L.; Ma, Y. Crystal structure prediction via particle-swarm optimization. Phys. Rev. B 2010, 82, 094116.

(25) Wang, Y.; Lv, J.; Zhu, L.; Ma, Y. CALYPSO: A method for crystal structure prediction. Comput. Phys. Commun. 2012, 183, 20632070.

(26) Kresse, G.; Furthmüller, J. Efficiency of ab-initio total energy calculations for metals and semiconductors using a plane-wave basis set. Comput. Mater. Sci. 1996, 6, 15-50.

(27) Kresse, G.; Joubert, D. From ultrasoft pseudopotentials to the projector augmented-wave method. Phys. Rev. B 1999, 59, 1758-1775.

(28) José, M. S.; Emilio, A.; Julian, D. G.; Alberto, G.; Javier, J.; Pablo, O.; Daniel, S.-P. The SIESTA method for ab initio order-N materials simulation. J. Phys.: Condens. Matter 2002, 14, 2745.

(29) Perdew, J. P.; Burke, K.; Ernzerhof, M. Generalized Gradient Approximation Made Simple. Phys. Rev. Lett. 1996, 77, 3865-3868.

(30) Postnikov, A.; Pagès, O.; Hugel, J. Lattice dynamics of the mixed semiconductors (Be, Zn) Se from first-principles calculations. Phys. Rev. B 2005, 71, 115206.
(31) Matthew, G. T.; Martin, T. D.; David, A. K. Simultaneous analysis of changes in long-range and short-range structural order at the displacive phase transition in quartz. J. Phys.: Condens. Matter 2000, 12, L723.

(32) Li, Q.; Zhou, D.; Zheng, W.; Ma, Y.; Chen, C. Global Structural Optimization of Tungsten Borides. Phys. Rev. Lett. 2013, 110, 136403.

(33) Kappera, R.; Voiry, D.; Yalcin, S. E.; Branch, B.; Gupta, G.; Mohite, A. D.; Chhowalla, M. Phase-engineered low-resistance contacts for ultrathin $\mathrm{MoS}_{2}$ transistors. Nat. Mater. 2014, 13, 11281134.

(34) Ross, N. L.; Shu, J.; Hazen, R. M. High-pressure crystal chemistry of stishovite. Am. Mineral. 1990, 75, 739-747.

(35) Nosé, S. A unified formulation of the constant temperature molecular dynamics methods. J. Chem. Phys. 1984, 81, 511-519.

(36) Topsakal, M.; Cahangirov, S.; Ciraci, S. The response of mechanical and electronic properties of graphane to the elastic strain. Appl. Phys. Lett. 2010, 96, 091912.

(37) Wang, G.; Si, M.; Kumar, A.; Pandey, R. Strain engineering of Dirac cones in graphyne. Appl. Phys. Lett. 2014, 104, 213107.

(38) Zeinalipour-Yazdi, C. D.; Christofides, C. Linear correlation between binding energy and Young's modulus in graphene nanoribbons. J. Appl. Phys. 2009, 106, 054318.

(39) Lee, C.; Wei, X.; Kysar, J. W.; Hone, J. Measurement of the elastic properties and intrinsic strength of monolayer graphene. Science 2008, 321, 385-388.

(40) Baron, M.; Stacchiola, D.; Ulrich, S.; Nilius, N.; Shaikhutdinov, S.; Freund, H.-J.; Martinez, U.; Giordano, L.; Pacchioni, G. Adsorption of $\mathrm{Au}$ and $\mathrm{Pd}$ atoms on thin $\mathrm{SiO}_{2}$ films: The role of atomic structure. J. Phys. Chem. C 2008, 112, 3405-3409.

(41) Tersoff, J.; Hamann, D. R. Theory and Application for the Scanning Tunneling Microscope. Phys. Rev. Lett. 1983, 50, 19982001.

(42) Wang, G.; Pandey, R.; Karna, S. P. Phosphorene oxide: stability and electronic properties of a novel two-dimensional material. Nanoscale 2015, 7, 524-531.

(43) Gupta, S. K.; He, H.; Banyai, D.; Si, M.; Pandey, R.; Karna, S. P. Effect of Si doping on the electronic properties of $\mathrm{BN}$ monolayer. Nanoscale 2014, 6, 5526-5531.

(44) Kumar, A.; Banyai, D.; Ahluwalia, P.; Pandey, R.; Karna, S. P. Electronic stability and electron transport properties of atomic wires anchored on the $\mathrm{MoS}_{2}$ monolayer. Phys. Chem. Chem. Phys. 2014, 16, 20157-20163.

(45) Gupta, S. K.; He, H.; Banyai, D.; Kandalam, A. K.; Pandey, R. Electron tunneling characteristics of a cubic quantum dot, $(\mathrm{PbS})_{32}$. J. Chem. Phys. 2013, 139, 244307.

(46) Román-Pérez, G.; Soler, J. M. Efficient implementation of a van der Waals density functional: application to double-wall carbon nanotubes. Phys. Rev. Lett. 2009, 103, 096102.

(47) Tao, J.; Luttrell, T.; Batzill, M. A two-dimensional phase of $\mathrm{TiO}_{2}$ with a reduced bandgap. Nat. Chem. 2011, 3, 296-300.

(48) Rawat, V.; Zakharov, D. N.; Stach, E. A.; Sands, T. D. Pseudomorphic stabilization of rocksalt $\mathrm{GaN}$ in TiN/GaN multilayers and superlattices. Phys. Rev. B 2009, 80, 024114.

(49) Chernozatonskii, L. A. A new class of $\mathrm{MO}_{2}$ dioxide nanotubes $(\mathrm{M}=\mathrm{Si}, \mathrm{Ge}, \mathrm{Sn}, \mathrm{Pb})$ composed of "square" lattices of atoms: Their structure and energy characteristics. JETP Lett. 2004, 80, 628-632.

(50) Artyukhov, V. I.; Chernozatonskii, L. A. Theoretical study of two-dimensional silica films. J. Phys. Chem. C 2010, 114, 9678-9684.

\section{NOTE ADDED IN PROOF}

The authors have recently become aware of the plane-wave density functional theory study suggesting that the tetragonal 2D silica structure (referred to as T-silica in our paper) can be basis for single-walled nanotubes of silica. ${ }^{49,50}$ 